\title{
Establishment of a biophysical model to optimize endoscopic targeting of magnetic nanoparticles for cancer treatment
}

This article was published in the following Dove Press journal:

International Journal of Nanomedicine

18 August 2017

Number of times this article has been viewed

\author{
Anjali A Roeth ${ }^{1, *}$ \\ loana Slabu',* \\ Martin Baumann² \\ Patrick H Alizai' \\ Maximilian Schmeding ${ }^{\prime}$ \\ Gernot Guentherodt ${ }^{3}$ \\ Thomas Schmitz-Rode ${ }^{2}$ \\ UIf P Neumann' \\ 'Department of General,Visceral \\ and Transplant Surgery, University \\ Hospital RWTH Aachen, ${ }^{2}$ Institute \\ of Applied Medical Engineering, \\ Helmholtz-Institute Aachen, RWTH \\ Aachen, Aachen, ${ }^{3}$ Institute of Physics \\ A, RWTH Aachen University, Aachen, \\ Germany \\ *These authors contributed equally \\ to this work
}

Correspondence: Anjali A Roeth Department of General, Visceral and Transplant Surgery, University Hospital RWTH Aachen, Pauwelsstrasse 30, 52074 Aachen, Germany

Tel +49 24I 8089500

Fax +49 24I 8082417

Email aroeth@ukaachen.de
Abstract: Superparamagnetic iron oxide nanoparticles (SPION) may be used for local tumor treatment by coupling them to a drug and accumulating them locally with magnetic field traps, that is, a combination of permanent magnets and coils. Thereafter, an alternating magnetic field generates heat which may be used to release the thermosensitively bound drug and for hyperthermia. Until today, only superficial tumors can be treated with this method. Our aim was to transfer this method into an endoscopic setting to also reach the majority of tumors located inside the body. To find the ideal endoscopic magnetic field trap, which accumulates the most SPION, we first developed a biophysical model considering anatomical as well as physical conditions. Entities of choice were esophageal and prostate cancer. The magnetic susceptibilities of different porcine and rat tissues were measured with a superconducting quantum interference device. All tissues showed diamagnetic behavior. The evaluation of clinical data (computed tomography scan, endosonography, surgical reports, pathological evaluation) of patients gave insight into the topographical relationship between the tumor and its surroundings. Both were used to establish the biophysical model of the tumors and their surroundings, closely mirroring the clinical situation, in which we could virtually design, place and evaluate different electromagnetic coil configurations to find optimized magnetic field traps for each tumor entity. By simulation, we could show that the efficiency of the magnetic field traps can be enhanced by 38 -fold for prostate and 8 -fold for esophageal cancer. Therefore, our approach of endoscopic targeting is an improvement of the magnetic drug-targeting setups for SPION tumor therapy as it holds the possibility of reaching tumors inside the body in a minimal-invasive way. Future animal experiments must prove these findings in vivo.

Keywords: drug targeting, simulation, SPION, prostate cancer, esophageal cancer

\section{Introduction}

The systemic application of a drug, especially a chemotherapeutic agent for tumor therapy, holds the major disadvantage of untargeted delivery. This is known to lead to serious side effects as the drug acts on other systems and healthy cells as well. Therefore, research is undertaken on local and targeted delivery of drugs. One approach is the use of superparamagnetic nanoparticles as vehicles, which bears two advantages: first, a high drug amount can be accumulated at the tumor site, and second, by the application of an alternating magnetic field, hyperthermia treatment is possible.

\section{Superparamagnetic nanoparticles}

Superparamagnetic iron oxide nanoparticles $(\mathrm{SPION})$ consist of magnetite $\left(\mathrm{Fe}_{3} \mathrm{O}_{4}\right)$ or maghemite $\left(\gamma-\mathrm{Fe}_{2} \mathrm{O}_{3}\right)$ with a size between 1 and $100 \mathrm{~nm} .{ }^{1}$ Due to their nanoscale size, they are able to react very strongly to a magnetic field, and do not show remanescence, 
that is, no magnetization after the field has been switched off. This is particularly important when used in vivo as they would agglomerate otherwise..$^{2-4}$

There are generally two ways to apply SPION for drug therapy and hyperthermia. The SPION can either be inserted directly into the tumor or a tumor-supplying artery with a syringe or a punch, or injected into a peripheral vein and then trapped at the tumor site with a magnetic field from outside. ${ }^{5-7}$ An additional alternating magnetic field may be used to generate heat in the tumor tissue through Néel and Brownian relaxation with the consequence that the thermosensitive layer melts and the drug is released at the tumor site. ${ }^{8}$ Nonetheless, for the development of our model, we focused on the accumulation of the SPION and did not deal with the application of the alternating magnetic field. The use of SPION for hyperthermia has been demonstrated already for different tumors (glioblastoma multiforme, prostate cancer, esophageal cancer) by Jordan et al who applied a suspension of nanoparticles directly into the tumor by punching. ${ }^{5}$ This approach is invasive and holds the risk of distributing cancer cells which might lead to metastases.

Therefore, a noninvasive enhancement at the region of interest after injecting the SPION into a peripheral vein and concentrating them at the tumor site is a promising alternative. Until today, the feasibility of this approach has been shown only for tumors near the surface as an extracorporeal magnetic field can be used for targeting purposes only on the tissue surface. ${ }^{9,10}$ Examples for the application of extracorporeal magnets can be found in the study by Widder et al who treated a sarcoma at a rat's tail, or the study by Alexiou who used pole shoes to treat tumors. ${ }^{6,11,12}$ However, the injection near the tumor without application of magnetic fields is not sufficient for SPION accumulation, because over $50 \%$ of SPION are cleared through the liver after a couple of minutes as shown by Lübbe et al. ${ }^{7}$

The targeting of the SPION and the local drug release remains an unsolved problem for tumors and organs located interior in the body. We hypothesize that a sufficient magnetic field to accumulate the SPION in internal tumors can be reached, if the magnet accumulating the nanoparticles is placed endoscopically in close neighborhood to the tumor. The ideal magnetic field for SPION retention at the tumor site is to be built like a trap with very high magnetic field gradients at the tumor site. Such a magnetic field can be configurated by an arrangement of electromagnetic coils designed according to the local situation. With an endoscopic approach, the electromagnetic coils are placed intraluminally through natural orifices such as the urethra or the mouth and the gastrointestinal tract. The size of the considered coils is chosen with respect to the endoscopy requirements and boundary conditions, which are well tolerated by the patients, that is, urinary catheters, rectoscopes or gastroscopes. In addition, safety and heat generation concerns have to be taken into account such as limiting the electric current to $50 \mathrm{~mA}$.

As examples of use, we chose prostate cancer and esophageal adenocarcinoma, both being endoluminal tumors that often require a multimodal treatment concept, that is, a combination of chemotherapy and other treatment plans such as radiation, hyperthermia or surgery. Prostate cancer was selected because of easy access due to the anatomical conditions as coil arrangements could be placed noninvasively in the urethra and the rectum and, therefore, the tumor could be reached from two sides. As a second example of use, we concentrated on adenocarcinoma of the esophagus as it metastasizes for the main part lymphogenic and therefore locally and only in advanced stages hematogenic with distant metastases, making it an ideal tumor entity for local drug treatment. Due to the large diameter of the esophagus, coil formations can be placed here easily as well.

Our aim was to find an ideal endoscopic magnetic field configuration for the above-mentioned tumor entities. Therefore, we first developed a biophysical model that considered anatomical as well as physical conditions. In a second step, we virtually designed and optimized different coil arrangements to find the coil configuration that best fulfilled our requirements, that is, very high magnetic field gradients at the tumor site.

\section{Materials and methods} Porcine and rat tissues

To obtain an accurate biophysical model of the target organs, the tumors and the surrounding tissue, we determined the magnetic characteristics (susceptibility) of different native tissues without SPION. For this endeavor, we used tissue samples of the relevant surrounding organs (esophagus, prostate, small intestine, large intestine, stomach, duodenum, liver, pancreas, lung, heart and muscle) from animal cadavers. As we did not work with living animals, no ethical approval was needed according to German law and in agreement with the State Agency for Nature, Environment and Consumer Protection, North Rhine-Westphalia, Germany. The animal cadavers were from animals from other approved animal experiments directly after euthanization (ethics committee of the LANUV [State Agency for nature, Environment and Consumer protection], North Rhine-Westphalia). These animal-handling procedures were performed according to the Guide for the Care and Use of Laboratory Animals of the National Institutes of Health and followed the guidelines of the Animal Welfare Act. All tissues were cryopreserved with fluid nitrogen immediately after explantation. Pretests 
had shown that the magnetic characteristics are not altered by this procedure (unpublished data). Aside from pigs (German Landrace), we chose rats (Wistar) as test animals to also search for differences in the magnetic characteristics between mammalian species.

\section{Measurement of the magnetic properties with a SQUID magnetometer}

The magnetic properties were measured with a superconducting quantum interference device (SQUID; Quantum Design, San Diego, CA, USA). This instrument measures extremely small changes in magnetic fields and, hence, the magnetic characteristics (ie, the susceptibility) of materials with a very high sensitivity. The susceptibility represents the degree to which a magnetic field is enhanced (paramagnetism) or extenuated (diamagnetism) within the respective material.

In order to perform the measurements under vacuum conditions, we sealed the tissues hermetically by use of glass nuclear magnetic resonance (NMR) tubes (Sigma Aldrich, St Louis, MO, USA) that were closed by melting both ends. The magnetic moments were measured at various magnetic field intensities at a temperature of $300 \mathrm{~K}$. The gradient $m$ given by

$$
m=\frac{\text { Magnetic moments }}{\text { Field intensity }}
$$

was determined by a least square fit. The susceptibility is then the gradient per volume.

$$
\begin{aligned}
\text { Susceptibility } & =\frac{\text { Magnetization }}{\text { Field intensity }} \\
& =\frac{\text { Magnetic moments }}{\text { Field intensity } \times \text { Volume }}=\frac{m}{\text { Volume }}
\end{aligned}
$$

\section{Evaluation of clinical data and establishment of biophysical models}

To test different coil configurations virtually, we established a biophysical model for each tumor entity that included biological (location, topography and surroundings, tumor volume) as well as physical (magnetic susceptibility) data. To closely mirror the pathophysiological setting of the two tumor entities, we evaluated computed tomography (CT) scans of 20 patients with the diagnosis of esophageal cancer and eight patients with the diagnosis of prostate cancer. The CT scans were analyzed with respect to tumor volumetry, location and topographical relationships. Volumetry was performed using OsiriX ${ }^{\circledR}$ software version 4.1.1. In addition, we analyzed the endoscopic data, surgical reports and postoperative pathological evaluation regarding tumor sizes.
Only patients with prostate cancer with a scoring of Gleason 7 and patients with esophageal cancer with a staging of T3 were included into this study to have a more uniform group. Ethics Committee of the Medical Faculty of the RWTH Aachen, Germany deemed no patient consent had to be obtained due to the retrospective nature of the descriptive and pseudonymized clinical evaluation.

\section{Simulations in MATLAB ${ }^{\circledR}$}

With the results of the measurement of the magnetic properties and the evaluation of the clinical data, we designed and established virtual biophysical models of the two tumor entities and their surrounding tissues in the programming environment MATLAB (The MathWorks Inc., Natick, MA, USA). The program MATLAB provides solutions of mathematical functions, here integrals, based on matrix manipulations. This enabled us to virtually design, place and evaluate magnetic field traps in the esophagus or the urethra and rectum, respectively. For the biophysical model, the organs were primarily defined and designed by geometrical figures and then changed according to the biological conditions, for example, by applying rotation and shifting matrices. The necessary boundary conditions were chosen according to details gained from the evaluation of clinical data, for example, diameter of the esophagus, size and position of the tumor and narrowing of the inner diameter at the tumor site due to compression by the tumor.

The magnetic field gradients were produced by a combination of different electromagnetic coils. We developed a programming routine in MATLAB which calculates and displays the magnetic field generated by any given coil configuration. The field strength was determined by the Biot-Savart law:

$$
\vec{H}=\frac{1}{4 . \pi} \oint \frac{d \vec{l} \times \vec{r}}{r^{3}}
$$

Here, $\overrightarrow{d l}$ is the infinitesimal length of a conductor carrying the electric current $I$, and $\vec{r}$ is the unit vector to specify the vector distance $r$ from the current to the field point.

Each electromagnetic coil consisted of 64 straight sections put together in a circle. The space around the coil configuration and the tumor was divided into nearly 25,000 cuboids. The program calculated the strength and direction of the resulting magnetic field in each cuboid. The resulting magnetic field was visualized in a logarithmic color scale for all cuboids in a certain plane. Figure 1 reveals the simulated field of a single coil in two different planes as an example.

For both tumor entities, a starting coil configuration was chosen on a physical basis. In the case of prostate cancer, 

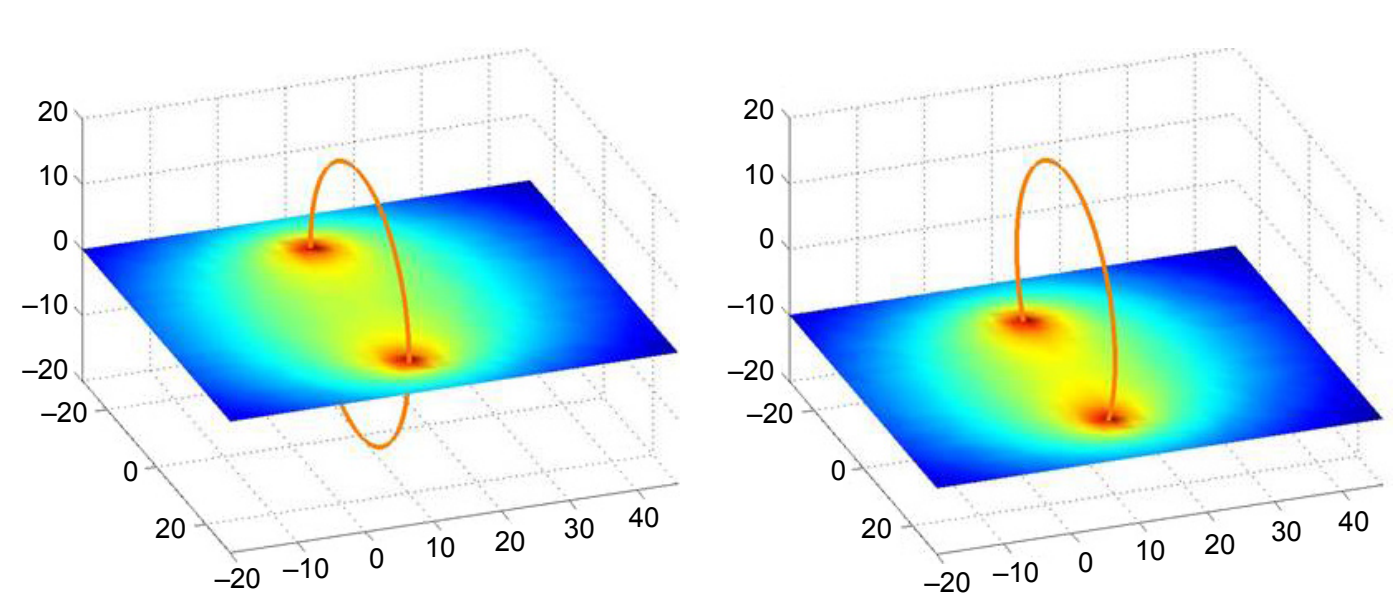

$2.0 \times 1 \mathrm{e}-4 \mathrm{~T}$

$1.2 \times 1 \mathrm{e}-4 \mathrm{~T}$

$7.5 \times 1 \mathrm{e}-5 \mathrm{~T}$

$4.5 \times 1 \mathrm{e}-5 \mathrm{~T}$

$2.7 \times 1 \mathrm{e}-5 \mathrm{~T}$

$1.7 \times 1 \mathrm{e}-5 \mathrm{~T}$

$1.0 \times 1 \mathrm{e}-5 \mathrm{~T}$

$6.1 \times 1 \mathrm{e}-6 \mathrm{~T}$

$3.7 \times 1 \mathrm{e}-6 \mathrm{~T}$

$2.3 \times 1 \mathrm{e}-6 \mathrm{~T}$

Figure I Magnetic field of a single coil in two different planes calculated by the developed programming routine in MATLAB ${ }^{\circledR}$.

Note: Unit of measurement is $\mathrm{cm}$.

the starting coil configuration consisted of five small coils in the urethra and one larger coil in the rectum. For esophageal cancer, a linear coil configuration of five coils in the esophagus was chosen.

The optimized coil configuration was found by stepwise convergence to the best result.

In the simulation, the parameters number, size, location and electric current of the coils were varied. The upper limit for each parameter was chosen with respect to safety concerns (eg, electric current under $50 \mathrm{~mA}$ ) as well as the size of the hollow organ where the coils were virtually placed in. For the urethra, an upper limit of $6 \mathrm{~mm}$ was set related to the size of urinary catheters; for the rectum, the upper limit was $25 \mathrm{~mm}$ (the size of a rectoscope) and for the esophagus $10 \mathrm{~mm}$ (size of a small gastroscope) as patients often develop esophageal stenosis due to the tumor.

In each step, one of the parameters was varied within the allowed range, while the others remained unchanged. The best result for this parameter was used in the following variations. For each variation, the profile and strength of the resulting magnetic field were determined.

For each configuration, an efficiency criterion was determined. The efficiency criterion is defined as the weighted sum over the magnetic field strengths at each point of the simulated area for a certain coil configuration. The weighting factor reflects the required magnetic field to build an ideal magnetic field trap, that is, high magnetic force at the tumor, steep gradient and no magnetic field outside of the tumor. It is specific for each tumor entity. For example, Figure 2 displays the weighting factor used for prostate cancer. As tumors in the prostate gland are often located in the posterior part of the gland, we chose an asymmetric distribution of the region with the highest weighting factor to ensure that the magnetic field is highest at the tumor site and has no major effect on the healthy surrounding tissue.

\section{Results}

\section{SQUID measurements reveal diamagnetic behavior of the tissues}

The magnetic properties of the target tissues and the surrounding organs were measured by SQUID magnetometry. Figure 3 reveals the sample holder consisting of a plastic tube in which the hermetically sealed capsules which contained the respective tissue are fixed with cotton wool from both sides. Cotton wool was chosen because of its low magnetic signal.

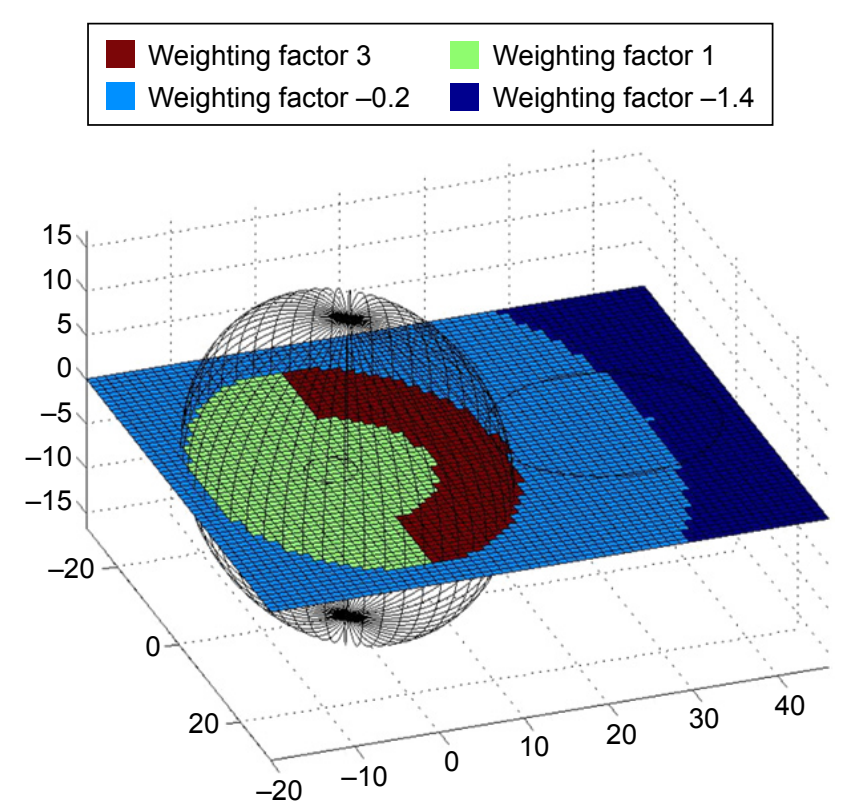

Figure 2 Weighting factors in different areas for prostate cancer: brown, tumor area; green, healthy prostate gland; light blue, surrounding area outside the prostate gland; and dark blue, region far away from tumor.

Note: Unit of measurement is $\mathrm{cm}$. 


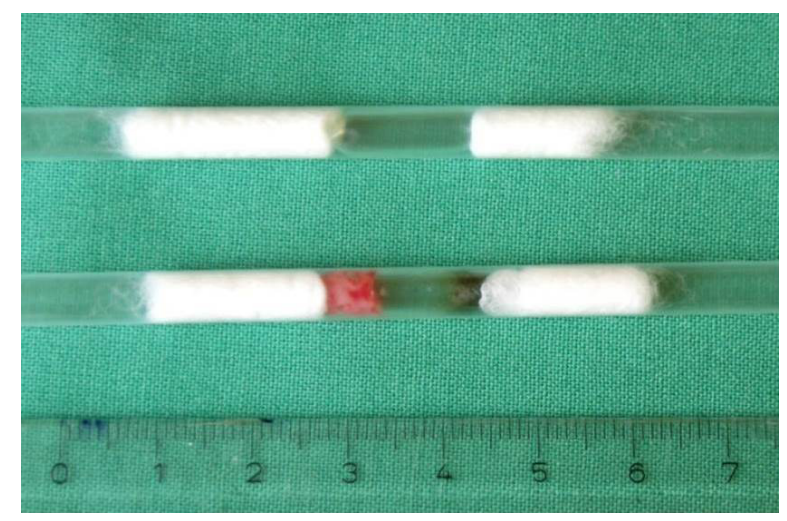

Figure 3 Hermetically sealed capsules with tissue, fixed with cotton wool and placed in plastic tubes.

Figure 4 depicts the dependence of the magnetic moments on the applied magnetic field strength for native porcine tissue (native rat tissue showed similar results) after background subtraction. A diamagnetic behavior was measured for all tissues regardless of the species. The magnetic susceptibilities entered the construction of the biophysical models.

\section{Evaluation of clinical data}

\section{and establishment of a biophysical model}

The evaluation and geometrical measurement of the CT scans of patients with adenocarcinoma of the esophagus or prostate cancer as well as surgical reports and postoperative pathological evaluation with measurement of the actual tumor size gave insight into the topographical relationships of the tumors and the different surrounding tissues. These findings

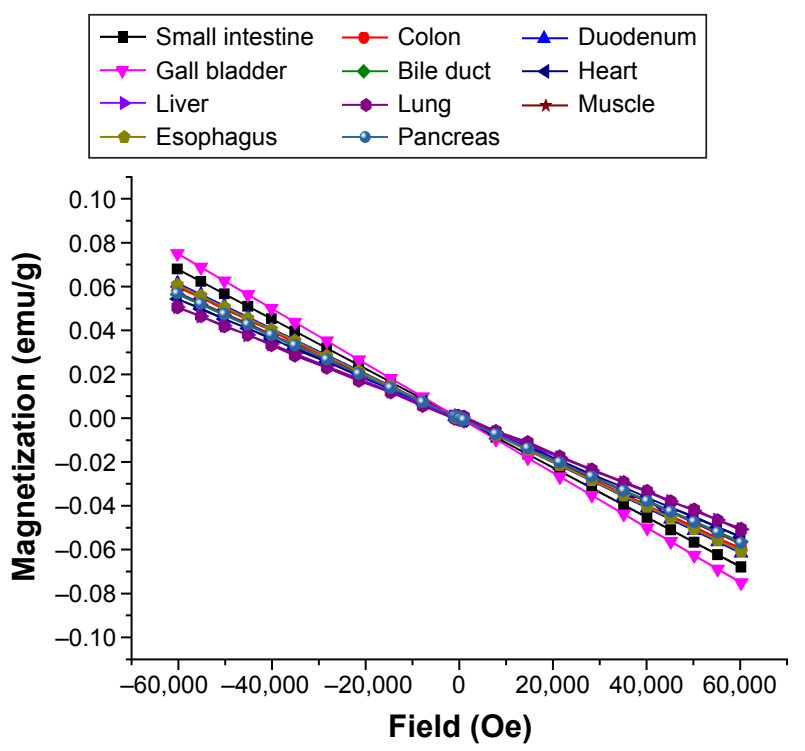

Figure 4 Magnetic moments of native porcine tissue dependent on applied magnetic field strength (conversion of Gaussian units to SI units: I emu $=10^{-3} \mathrm{Am}^{2} ; 1 \mathrm{Oe}=\frac{10^{3}}{4 \pi} \frac{\mathrm{A}}{\mathrm{m}}$ ). were incorporated in the biophysical models to define the volume, in which the SPION needed to be targeted, as well as the diameter of the orifices where the magnetic coils could be installed. For the tumor-bearing prostate, an average size of $3.54 \pm 0.61 \mathrm{~cm} \times 4.76 \pm 0.39 \mathrm{~cm} \times 3.5 \pm 0.57 \mathrm{~cm}$ was determined. In case of esophageal cancer, the target volume has to be larger than the tumor volume itself as patients with a tumor staging of $\mathrm{T} 3$ often already have lymphatic metastases (13 out of 20 in our patients). Therefore, the target volume was defined to be $8.5 \times 8.5 \times 3 \mathrm{~cm}$ as this would include the tumor and the local lymphatic metastases for all tumors investigated. One also has to take into account that the lumen of the esophagus is often narrowed by tumors. Hence, the maximal inner diameter of the esophagus was set to $1 \mathrm{~cm}$.

With the magnetic properties and the sizes and topographical relationships between the tumors and their neighboring structures, we established a biophysical model in the programming environment MATLAB. To visualize the two selected models, Figure 5 displays the borders of the prostate, the urethra and the rectum as well as the esophagus, the tumor and the tumor stenosis. Also, the starting coil configurations regarding physical considerations are displayed.

\section{Simulations in MATLAB}

By variation of all parameters, a coil configuration was found that produced an optimized magnetic field trap within the target volume. Starting with the configurations shown in Figure 5, an optimized setup could be reached as displayed in Figure 6 which best fulfilled the requirements of a very steep magnetic field gradient at the tumor site. The resulting magnetic field is depicted likewise in two different intersecting planes for the prostate as well as for the esophagus.

In case of the prostate, the highest efficiency criterion could be achieved with five coils in the urethra and no coil in the rectum. As the distribution of the tumor is not symmetric around the urethra, the result was a unique coil arrangement with two coils paralleled in the urethra as revealed in Figure 6. With optimized geometry, an improvement in targeting efficiency of a factor of 38 could be achieved. For the esophagus, the starting coil arrangement already made use of the results for prostate cancer. Still, an 8-fold enhancement of the efficiency criterion could be achieved for this entity as well.

\section{Discussion}

The use of magnetic nanoparticles for local cancer treatment is a highly promising approach toward an individualized therapy for many tumors because the chemotherapeutic agent acts locally. ${ }^{13}$ It helps to reduce the serious side effects of any cytotoxic therapy. In addition, it allows for hyperthermia 
A

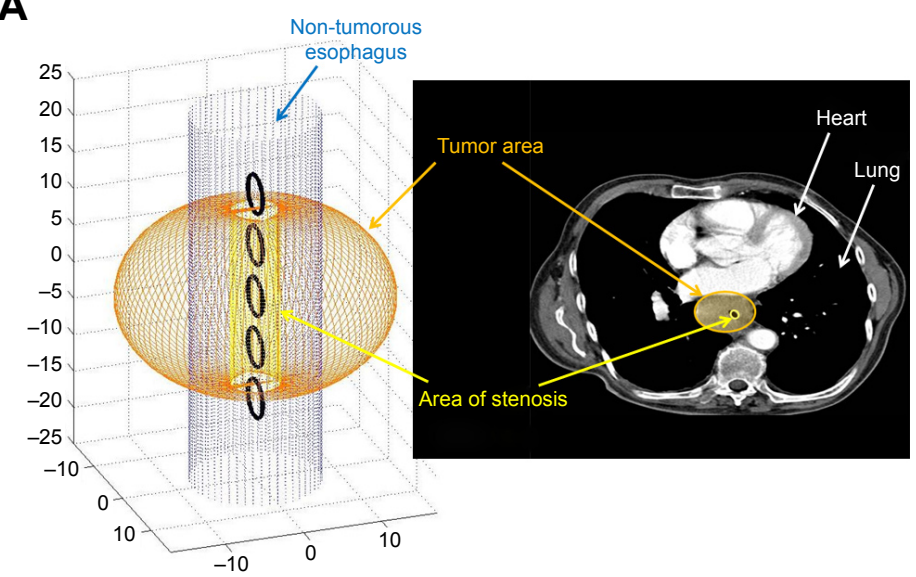

B

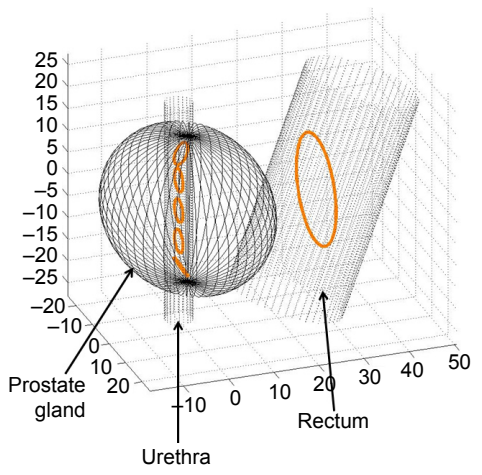

Figure 5 Biophysical model in MATLAB ${ }^{\circledR}$ for adenocarcinoma of the esophagus and CT scan $(\mathbf{A})$, and prostate cancer with borders of the prostate, the urethra and the rectum (B).

Abbreviation: CT, computed tomography.

treatment of the tumor, a therapeutic option that helps to reduce tumors in a lot of entities. ${ }^{14}$

Our approach of endoscopic targeting is an improvement of the known use of magnetic nanoparticles in tumor therapy as it holds the possibility of reaching tumors inside the body in a minimally invasive way. Most other groups apply the SPION by punching holes into the tumor or by injection into a tumor-supplying artery. ${ }^{12,15,16}$ The magnetic field to target the tumors is achieved by an extracorporeal coil configuration; therefore, only tumors close to the surface of the body

A
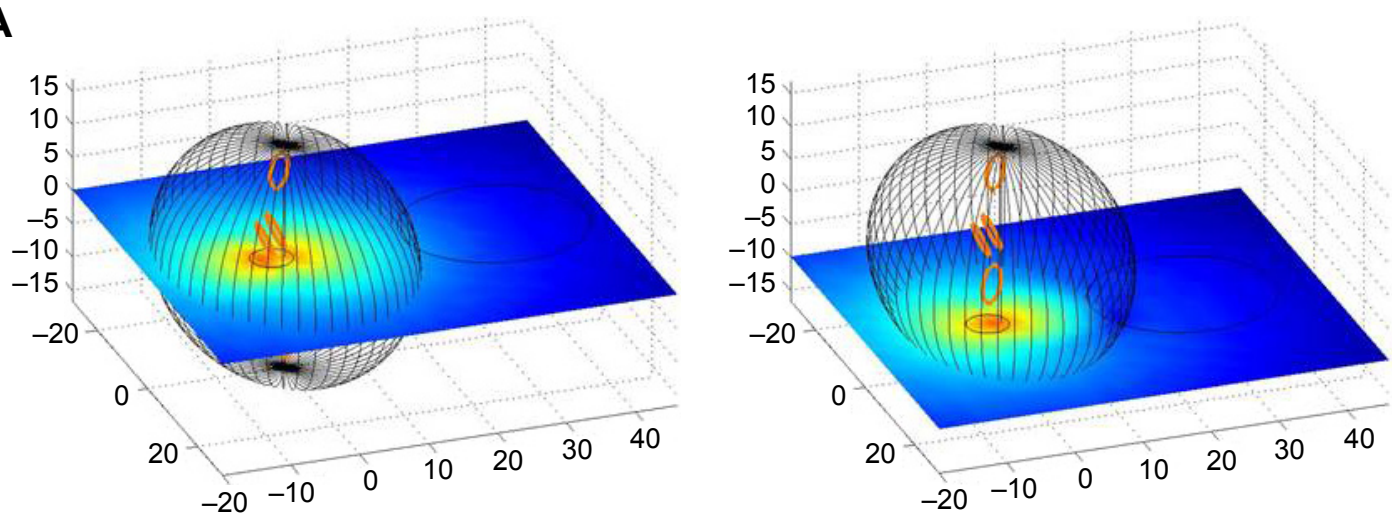

$2.0 \times 1 \mathrm{e}-4 \mathrm{~T}$

$1.2 \times 1 \mathrm{e}-4 \mathrm{~T}$

$7.5 \times 1 \mathrm{e}-5 \mathrm{~T}$

$4.5 \times 1 \mathrm{e}-5 \mathrm{~T}$

$2.7 \times 1 \mathrm{e}-5 \mathrm{~T}$

$1.7 \times 1 \mathrm{e}-5 \mathrm{~T}$

$1.0 \times 1 \mathrm{e}-5 \mathrm{~T}$

$6.1 \times 1 \mathrm{e}-6 \mathrm{~T}$

$3.7 \times 1 \mathrm{e}-6 \mathrm{~T}$

$2.3 \times 1 \mathrm{e}-6 \mathrm{~T}$

\section{B}

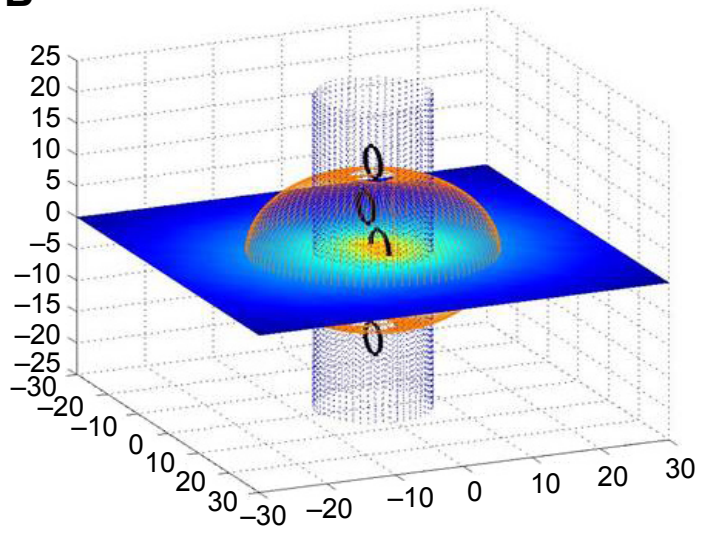

$2.0 \times 1 \mathrm{e}-4 \mathrm{~T}$

$1.2 \times 1 \mathrm{e}-4 \mathrm{~T}$

$7.5 \times 1 \mathrm{e}-5 \mathrm{~T}$

$4.5 \times 1 \mathrm{e}-5 \mathrm{~T}$

$2.7 \times 1 \mathrm{e}-5 \mathrm{~T}$

$1.7 \times 1 \mathrm{e}-5 \mathrm{~T}$

$1.0 \times 1 \mathrm{e}-5 \mathrm{~T}$

$6.1 \times 1 \mathrm{e}-6 \mathrm{~T}$

$3.7 \times 1 \mathrm{e}-6 \mathrm{~T}$

$2.3 \times 1 \mathrm{e}-6 \mathrm{~T}$

Figure 6 Optimized coil configuration with resulting magnetic field for prostate cancer (A) and esophageal cancer (B). Note: Unit of measurement is $\mathrm{cm}$. 
may be reached., ${ }^{9,10}$ Until today, the only way to treat tumors inside the body is with magnetic stents or implants, thus involving a surgical procedure ${ }^{17} \mathrm{We}$ were able to prove the feasibility of our endoscopic approach and to optimize it for a biophysical model of two different settings, prostate cancer and esophageal cancer.

\section{Measurement of the magnetic susceptibility with a SQUID}

For our biophysical model, the measurement of the magnetic susceptibility was essential in order to determine the resulting magnetic field of the different coil arrangements. To our knowledge, there is no study that measures the magnetic susceptibility of tissues quantitatively. Nonetheless, there are many papers in the field of medical physics or radiology that measure the magnetic susceptibility qualitatively with regard to the behavior in a magnetic field during magnetic resonance imaging (MRI). ${ }^{18-21}$

In essence, the measurement of the magnetization of a tissue specimen depending on the applied magnetic field and therefore the quantitative determination of the magnetic characteristics was performed for the first time. All tissues show diamagnetic properties due to the high water content. Still, we were able to identify differences between the different tissues. They seem to be dependent on the tissues content of hemoglobin and myoglobin, that is, less diamagnetic behavior, and therefore a shift toward paramagnetism for muscles and parenchymatous organs such as the liver. Future experiments will have to address this aspect more specifically.

As human tissues are scarce, we investigated two mammalian species, rats and pigs, to draw conclusions regarding human tissues. Tissues of both species behave alike in the magnetic field, so it seems justified to use their magnetic susceptibilities in the model of human cancer, too. Further experiments must prove the comparability of animal tissues with human tissue in detail.

\section{Biophysical model and simulations}

On the basis of the data from the SQUID analysis as well as the investigation of the geometric-topographical setting (CT scan, endoscopic data, surgical reports and postoperative pathological evaluation), we established biophysical models of prostate and esophageal cancer with their respective surroundings in MATLAB. In these models, we were able to calculate the strength and orientation of the resulting magnetic field of any given coil configuration.

The crucial part for an effective endoscopic targeting of the SPION and, therefore, an effective tumor treatment is finding the ideal magnetic field trap for each tumor. As we plan to treat the patient without narcosis endoscopically, the diameters of the magnetic coil arrangements have to be chosen according to corresponding diameters used in clinical routine. Therefore, the maximum size the electromagnetic coil configuration could achieve was set according to clinical practice (urethra $6 \mathrm{~mm}$, rectum $25 \mathrm{~mm}$ and esophagus $10 \mathrm{~mm}$ ) in our simulation.

The present biophysical model with the found encouraging results for the coil configuration can be transferred into an animal model of cancer as a next step. Thereby, other factors are likely to influence the targeting efficiency even more positively as the microscopical anatomy of the tumor and the blood vessels due to angiogenesis will have an additional targeting effect since the velocity of the blood is lower. An additional helpful characteristic of neo-vessels of tumors for targeting is the enhanced permeability and retention (EPR) effect. ${ }^{22}$ As opposed to healthy vessels, tumor neo-vessels are more permeable for macromolecules and nanoparticles due to less distinct endothelial barriers. This is important for the accumulation of SPION in the tumor-surrounding tissues. ${ }^{23}$

The MATLAB programming routine developed to determine the magnetic field of any coil arrangement holds a high variability regarding geometry and physics of the respective anatomical conditions. Hence, it can be used for different tumor entities and settings as well as to find the optimal coil configuration producing the magnetic field trap. As the model is very flexible, it may even be used for personalized therapy in the future. The model can be modified for each patient individually with respect to CT, MRI and endosonographic data, in order to find the optimal coil arrangement to produce a magnetic field trap.

Endoscopic targeted delivery of drugs by use of magnetic nanoparticles offers a huge spectrum of applications for different kinds of tumors besides prostate and esophageal cancers. Delivery of drugs other than chemotherapeutic agents is possible, too. ${ }^{24}$ In addition, the endoscopic magnetotargeted delivery can be combined with immunologicaltargeted delivery by linking with tumor-specific antibodies. ${ }^{25}$ Hereby, the SPION may first be guided to the tumor site with magnetic forces and thereafter target even more specifically only a certain cell type in the tumor microenvironment.

\section{Conclusion}

In conclusion, we were able to establish biophysical models of the tumor and its surroundings in two different tumor environments that closely mirrored the actual settings in patients. With these models, it was possible to virtually 
design, build and test different coil configurations. Their resulting magnetic field produces the optimal magnetic field trap in order to target the magnetic nanoparticles at the region of interest. Future animal experiments must prove the performance in vivo.

\section{Acknowledgments}

This project was supported by the START grant of the Medical Faculty of the RWTH Aachen University. The authors thank Ellen Krott and Jolande Fooken for help with the animal experiments.

\section{Disclosure}

The authors report no conflicts of interest in this work.

\section{References}

1. Häfeli UO, Chastellain M. Magnetic nanoparticles as drug carriers. In: Torchilin VP, editor. Nanoparticles as Drug Carriers. London: Imperial College Press; 2006.

2. Wang YX, Hussain SM, Krestin GP. Superparamagnetic iron oxide contrast agents: physicochemical characteristics and applications in MR imaging. Eur Radiol. 2001;11(11):2319-2331.

3. Gaur U, Sahoo SK, De TK, Ghosh PC, Maitra A, Ghosh PK. Biodistribution of fluoresceinated dextran using novel nanoparticles evading reticuloendothelial system. Int J Pharm. 2000;202(1-2):1-10.

4. Bulte JW, Duncan ID, Frank JA. In vivo magnetic resonance tracking of magnetically labeled cells after transplantation. J Cereb Blood Flow Metab. 2002;22(8):899-907.

5. Jordan A, Maier-Hauff K, Wust P, Rau B, Johannsen M. Thermotherapie mit Nanopartikeln. Onkologie. 2007;13:894-902.

6. Widder KJ, Morris RM, Poore GA, Howard DP, Senyei AE. Selective targeting of magnetic albumin microspheres containing low-dose doxorubicin: total remission in Yoshida sarcoma-bearing rats. Eur $J$ Cancer Clin Oncol. 1983;19(1):135-139.

7. Lübbe AS, Bergemann C, Riess H, et al. Clinical experiences with magnetic drug targeting: a phase I study with 4'-epidoxorubicin in 14 patients with advanced solid tumors. Cancer Res. 2001;56(20):4686-4693.

8. Reeves DB, Weaver JB. Approaches for modeling magnetic nanoparticle dynamics. Crit Rev Biomed Eng. 2014;42(1):85-93.

9. Alexiou C, Diehl D, Henninger P, et al. A high field gradient magnet for magnetic drug targeting. IEEE Trans Appl Supercond. 2006;16(2): $1527-1530$.

10. Gleich B, Hellwig N, Bridell H, et al. Design and evaluation of magnetic fields for nanoparticle drug targeting in cancer. IEEE Trans Nanotechnol. 2007;6(2):164-169.
11. Alexiou C. Nanomedicine. Innovative applications in medicine. HNO. 2013;61(3):197-201.

12. Lyer S, Tietze R, Jurgons R, et al. Visualisation of tumour regression after local chemotherapy with magnetic nanoparticles - a pilot study. Anticancer Res. 2010;30(5):1553-1557.

13. Mikhaylov G, Vasiljeva O. Promising approaches in using magnetic nanoparticles in oncology. Biol Chem. 2011;392(11):955-960.

14. Rao W, Deng ZS, Liu J. A review of hyperthermia combined with radiotherapy/chemotherapy on malignant tumors. Crit Rev Biomed Eng. 2010;38(1):101-116.

15. Maier-Hauff K, Ulrich F, Nestler D, et al. Efficacy and safety of intratumoral thermotherapy using magnetic iron-oxide nanoparticles combined with external beam radiotherapy on patients with recurrent glioblastoma multiforme. J Neurooncol. 2011;103(2):317-324.

16. Jordan A, Scholz R, Maier-Hauff K, et al. Presentation of a new magnetic field therapy system for the treatment of human solid tumors with magnetic fluid hyperthermia. J Magn Magn Mater. 2001;225:118-126.

17. Wahajuddin, Arora S. Superparamagnetic iron oxide nanoparticles: magnetic nanoplatforms as drug carriers. Int J Nanomedicine. 2012; 7:3445-3471.

18. Nörenberg D, Ebersberger HU, Walter T, et al. Diagnosis of calcific tendonitis of the rotator cuff by using susceptibility-weighted MR imaging. Radiology. 2016;278(2):475-484.

19. Kakeda S, Futatsuya K, Ide S, et al. Improved detection of cortical gray matter involvement in multiple sclerosis with quantitative susceptibility mapping. Acad Radiol. 2015;22(11):1427-1432.

20. Li X, Harrison DM, Liu H, et al. Magnetic susceptibility contrast variations in multiple sclerosis lesions. J Magn Reson Imaging. 2016;43(2): 463-473.

21. Kiricuta IC Jr, Simplăceanu V. Tissue water content and nuclear magnetic resonance in normal and tumor tissues. Cancer Res. 1975;35(5): 1164-1167.

22. Maeda H, Sawa T, Konno T. Mechanism of tumor-targeted delivery of macromolecular drugs, including the EPR effect in solid tumor and clinical overview of the prototype polymeric drug SMANCS. J Control Release. 2001;74(1-3):47-61.

23. Torchilin VP. Introduction: nanocarriers for drug delivery: needs and requirements. In: Torchilin VP, editor. Nanoparticles as Drug Carriers. London: Imperial College Press; 2006.

24. Tadayon A, Jamshidi R, Esmaeili A. Delivery of tissue plasminogen activator and streptokinase magnetic nanoparticles to target vascular diseases. Int J Pharm. 2015;495(1):428-438.

25. Bouras A, Kaluzova M, Hadjipanayis CG. Radiosensitivity enhancement of radioresistant glioblastoma by epidermal growth factor receptor antibody-conjugated iron-oxide nanoparticles. J Neurooncol. 2015;124(1):13-22.
International Journal of Nanomedicine

\section{Publish your work in this journal}

The International Journal of Nanomedicine is an international, peerreviewed journal focusing on the application of nanotechnology in diagnostics, therapeutics, and drug delivery systems throughout the biomedical field. This journal is indexed on PubMed Central, MedLine, CAS, SciSearch $\AA$, Current Contents $\AA /$ Clinical Medicine,

\section{Dovepress}

Journal Citation Reports/Science Edition, EMBase, Scopus and the Elsevier Bibliographic databases. The manuscript management system is completely online and includes a very quick and fair peer-review system, which is all easy to use. Visit http://www.dovepress.com/ testimonials.php to read real quotes from published authors. 